\title{
Study of a test methodology to assess potential drift generated by air-assisted sprayers
}

\author{
Paolo Balsari, Paolo Marucco, Claudio Bozzer, Mario Tamagnone \\ Dipartimento di Scienze Agrarie Forestali e Alimentari, DiSAFA, Università di Torino, Italy
}

\begin{abstract}
During pesticide application spray drift may cause diffuse pollution phenomena in the environment. In the last years the European Union, through the Directive on the sustainable use of pesticides (128/2009 $\mathrm{EC})$, has recommended the adoption of measures enabling to prevent spray drift. Among these measures, the adoption of buffer zones beside the sprayed fields requires to consider different widths for these no spray zones according to the amount of spray drift generated by the spraying equipment used for application. It is therefore necessary to classify the different sprayer models according to drift risk. For what concerns the sprayers used on arboreal crops, in order to make this classification in a simple and quick way as it was already proposed for the field crop sprayers (ISO FDIS 22369-3), a study was started aimed at defining a methodology to assess potential drift produced by the different sprayer models in absence of wind, using ad hoc test benches. On the basis of the positive first experimental data obtained, a first proposal for a new ISO standard methodology was prepared.
\end{abstract}

\section{Introduction}

The European Directive 128/2009 EC on sustainable use of pesticides prescribes to use the most efficient spraying equipment in order to prevent environmental contamination risks, especially those related to aquatic organisms. Prevention of spray drift is therefore a key aspect, as the dispersion of part of the droplets sprayed on crops outside the target field may cause not negligible contamination risks for the surrounding environment and even for bystanders. On the other hand, the recent amendment of the Machinery Directive (127/2009 EC) requires that all new sprayers comply with a set of technical fea-

Correspondence: Paolo Marucco, Dipartimento di Scienze Agrarie Forestali e Alimentari, DiSAFA, Università di Torino, via L. da Vinci 44, 10095 Grugliasco (T0), Italy

Tel. +39.011.6708599 - Fax: +39.011 .2368599 .

E-mail: paolo.marucco@unito.it

Key words: sprayer, drift, classification, test bench.

(C) Copyright P. Balsari et al., 2013

Licensee PAGEPress, Italy

Journal of Agricultural Engineering 2013; XLIV(s2):e69

doi:10.4081/jae.2013.s2.e69

This article is distributed under the terms of the Creative Commons Attribution Noncommercial License (by-nc 3.0) which permits any noncommercial use, distribution, and reproduction in any medium, provided the original author(s) and source are credited. tures which are relevant to guarantee the environmental safeguard in spray application.

In recent years several European countries have already adopted some measures to prevent risks of environmental contamination with pesticides, establishing buffer zones along water courses and sensitive areas. Generally, the width of the buffer zone is defined according to the type of plant protection product applied (toxicity, dose), to the characteristics of the area adjacent to the sprayed field (e.g. water courses, urban areas, other sensitive crops) and to the features of the spraying equipment used.

Classification of sprayers according to drift risk is therefore necessary in order to modulate the width of buffer zones in function of the sprayer type used. Actually ISO standard 22369 defines the classes of drift reduction with respect to a reference sprayer and refers to ISO standard 22866 for the assessment of spray drift in the field. This methodology, however, is rather complex, reproducibility of results is poor and results are strictly related to the agro-environmental context in which tests are carried out.

As there is a huge number of types and configurations of air-assisted sprayers for arboreal crops, their classification applying ISO 22866 test methodology would be long and expensive. Moreover, it should be repeated in each country/region taking into account the most common characteristics of the vineyards/orchards (layout, training system, plant size, etc.). Aim of the present study was therefore to develop a simpler methodology for assessing the potential drift generated by airassisted sprayers for arboreal crops in absence of wind.

\section{Materials and methods}

Tests were made using two different air-assisted sprayers: one for orchards (Nobili Oktopus 1000, equipped with 7+7 nozzles, radial fan and multiple hoses to convey the air to the air spouts positioned in correspondence of the nozzles) and one for vineyards (Dragone k2 500, equipped with 4+4 nozzles, axial fan and tower shaped air conveyor). Each sprayer was tested using either conventional hollow cone nozzles or air induction flat fan nozzles (Table 1). In all trials a forward speed of $6 \mathrm{~km} / \mathrm{h}$ was adopted and 5 replicates were made.

Volume application rate for the orchard sprayer was 1000 Vha, assuming to operate with a distance between rows of $4 \mathrm{~m}$, while for the vineyard sprayer it was $340 \mathrm{l} / \mathrm{ha}$, assuming to operate with a distance between rows of $2.5 \mathrm{~m}$.

Tests were carried out in absence of wind (maximum wind velocity $<0.5 \mathrm{~m} / \mathrm{s}$ ), operating the sprayer along a track made of concrete $50 \mathrm{~m}$ long and $3 \mathrm{~m}$ wide, in order to minimise the effects of eventual jerks on spray distribution. On the right side of the track, perpendicular to the sprayer forward direction, four arrays of Petri dishes were placed. In each array the first sampler was positioned at $2 \mathrm{~m}$ distance from the centre of the sprayer, the following samplers were positioned every one meter until $20 \mathrm{~m}$ distance from the centre of the sprayer (Figure 1A). The first array of Petri dishes was left always uncovered while the 
other three arrays were placed in ad hoc test benches designed at DiSAFA - University of Torino (Balsari et al., 2007). These test benches are provided with a sliding cover that is automatically activated by the tractor pass through a pneumatic system. In practice, a tractor mounted appendix hits a vertical pole that is linked to the mechanism of the sliding covers. Position of the vertical pole was established in such a way that test benches were uncovered after the sprayer pass when the spraying nozzles were, respectively, at distances of 2,4 and $6 \mathrm{~m}$ with respect to the benches (Figure 1B).

The system of samplers therefore enabled to assess to projection of the spray jet on the permanently uncovered Petri dishes and to evaluate, on the Petri dishes revealed only after the sprayer pass, the fraction of droplets that remain suspended in the air after the sprayer pass and then fall down to the ground. The latter represents the part of droplets more prone to drift as they can be blown out of the treated area by environmental wind.

Tests were carried out spraying a water solution of yellow tracer Tartrazine E $102(10 \% \mathrm{v} / \mathrm{v})$. Amount of spray deposits collected on plastic Petri dishes (15 $\mathrm{cm}$ diameter) were measured in laboratory by means of spectrophotometric analysis.

\section{Results}

Test results pointed out that the orchard sprayer is featured by a longer spray jet range (about $20 \mathrm{~m}$ distance from the centre of the sprayer) with respect to the vineyard sprayer, whose spray jet range is limited to about 14 metres from the sprayer centre (Figure 2). Profiles of spray jet range registered in the five replications of each test presented a good reproducibility in terms of sum of spray deposits registered at the difference sampling distances (Table 2).

Use of air induction nozzles on the orchard sprayer did not have a significant impact on the profile of the spray jet range (Figure 3A) while it reduced consistently the length of the spray jet range for the vineyard sprayer; in this latter case spray deposits were concentrated at 4-5 $\mathrm{m}$ from the centre of the sprayer (Figure 3B).

The spray deposits detected on the samplers positioned along the test benches resulted lower with respect to those measured on the permanently uncovered samplers. Deposits decreased according to the distance of the test bench from the point of activation of the sliding covers. This fact resulted more evident when air induction nozzles were used, either on orchard or on vineyard sprayers (Figures 4 and 5).

\section{Discussion}

On the basis of the results obtained a method for calculating a drift potential index (DPI) for each thesis examined was studied. As a first step, it was considered necessary to estimate the amount of spray recovered on the permanently uncovered samplers with respect to the

Table 1. Sprayers and operative parameters examined in the trials.

\begin{tabular}{lccccc} 
Test & Sprayer model & Nozmles & $\begin{array}{c}\text { Pressure } \\
(\mathrm{MPa})\end{array}$ & $\begin{array}{c}\text { Sprayer flow rate } \\
(\text { I/min) }\end{array}$ & $\begin{array}{c}\text { Fan air flow rate } \\
(\mathrm{m3} / \mathrm{h})\end{array}$ \\
\hline 1 & Nobili Oktopus & Teejet TXB 8004 & 1.0 & 40.3 & 14000 \\
2 & Nobili Oktopus & Teejet AI 11004 & 1.0 & 40.3 & 14000 \\
\hline 3 & Dragone k2 500 & Albuz ATR yellow & 1.0 & 8.4 & 20000 \\
4 & Dragone k2 500 & Lechler ID 12002 & 0.5 & 8.4 & 20000 \\
\hline
\end{tabular}

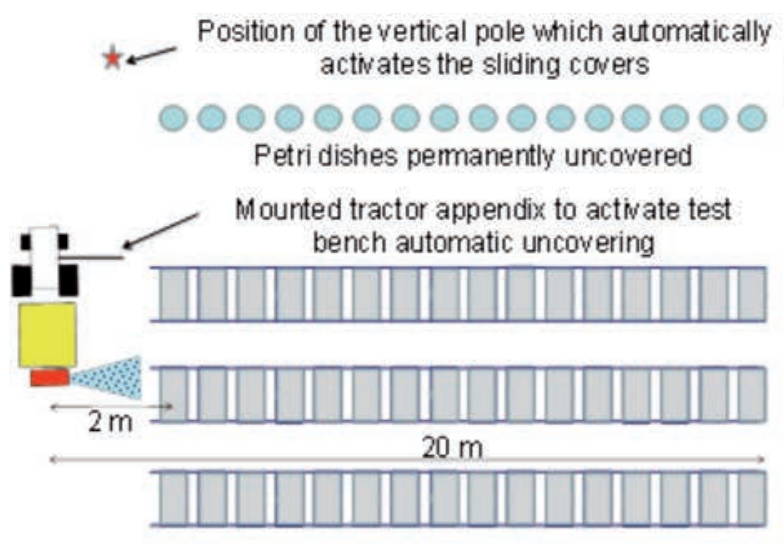

Test benches equipped with siding covers

A

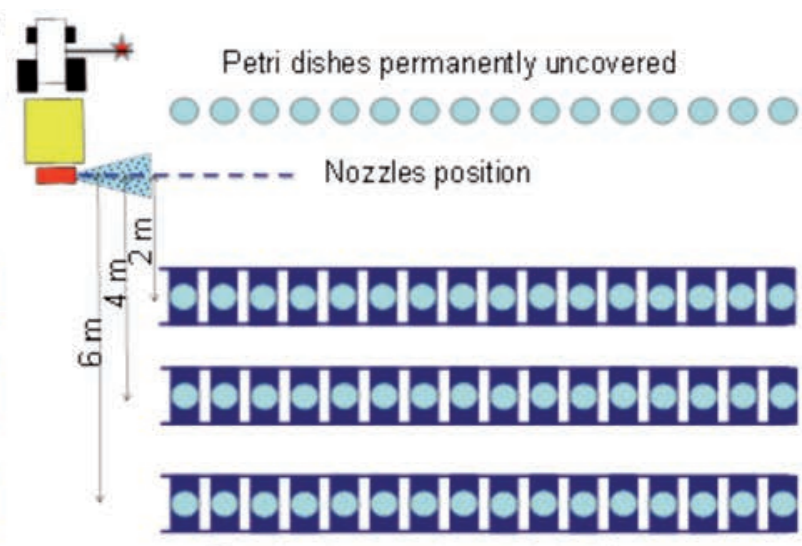

Test benches equipped with sliding covers

B

Figure 1. Scheme of samplers disposal to assess the range of the spray jet generated by the sprayer (permanently exposed collectors) and of the test benches disposal to detect the spray deposits which fall on the ground after the sprayer pass. A) Situation while the sprayer is passing besides the test covered benches; B) Just after the sprayer pass the test benches are uncovered and the samplers are revealed. 
Table 2. Values of the sum of spray deposits $(\mu 1 / \mathrm{cm} 2)$ obtained on the Petri dishes permanently exposed, measured in each of the five replicates made for each test (see Table 1) and coefficient of variation (CV) between the replicates.

\begin{tabular}{lcccccccc} 
Test & Rep 1 & Rep 2 & Rep 3 & Rep 4 & Rep 5 & Mean & \\
1 & 8.4 & 10.0 & 8.0 & 8.0 & 8.2 & 8.5 & $10 \%$ & 9.5 \\
2 & 9.9 & 9.2 & 10.6 & 9.1 & 8.5 & 1.8 & $11 \%$ \\
\hline 3 & 1.7 & 1.9 & 1.9 & 1.5 & 2.0 & 3.5 & 2.8 & $24 \%$ \\
4 & 2.9 & 3.2 & 1.7 & 2.8 & 3.5 & \\
\hline
\end{tabular}

amount sprayed by the machine. Therefore, taking into account the operative parameters adopted, for each thesis the amount of liquid sprayed per $\mathrm{cm}$ of advancing was calculated according to the following equation [1]:

$$
\mathrm{E}=\mathrm{Q} / \mathrm{v}
$$

where:

$\mathrm{E}$ is the amount of liquid sprayed per $\mathrm{cm}$ of advancing (expressed in $\mu \mathrm{l} / \mathrm{cm})$

Q is the total sprayer flow rate on one side of the machine (expressed in $\mu \mathrm{l} / \mathrm{s}$ );

$\mathrm{v}$ is the sprayer forward speed (expressed in $\mathrm{cm} / \mathrm{s}$ ).

The percentage of liquid recovered on the Petri dishes permanently exposed with respect to the amount sprayed was then calculated through the following equation [2]:

$$
\mathrm{RR}=\left[\sum \mathrm{D}_{\mathrm{i}} \times 100 / \mathrm{E}\right] \times 100
$$

where:

RR is the percentage of liquid recovered on the samplers;

$D_{i}$ is the spray deposit $(\mu 1 / \mathrm{cm} 2)$ registered at every sampling distance $i$; 100 is the distance (expressed in $\mathrm{cm}$ ) between two adjacent samplers.

In order to simplify the test method, concerning the spray deposits collected on the test benches, it was chosen to consider just the ones measured on the test bench positioned at $4 \mathrm{~m}$ distance from the nozzles when the bench was uncovered.

On the basis of the data registered on the test benches potential drift (DP) was calculated through the following equation [3]:

$$
\mathrm{DP}=\sum \mathrm{Si} \times \mathrm{d}_{\mathrm{i}} \times 100
$$

where:

DP is the potential drift;

$\mathrm{S}_{\mathrm{i}}$ is the spray deposit $(\mu \mathrm{l} / \mathrm{cm} 2)$ measured at each sampling distance $\mathrm{i}$ along the test bench;

$\mathrm{d}_{\mathrm{i}}$ is the sampling distance (expressed in $\mathrm{cm}$ ) of each sampler i from the sprayer centre.

Finally, thanks to the parameters calculated in the equations [2] and

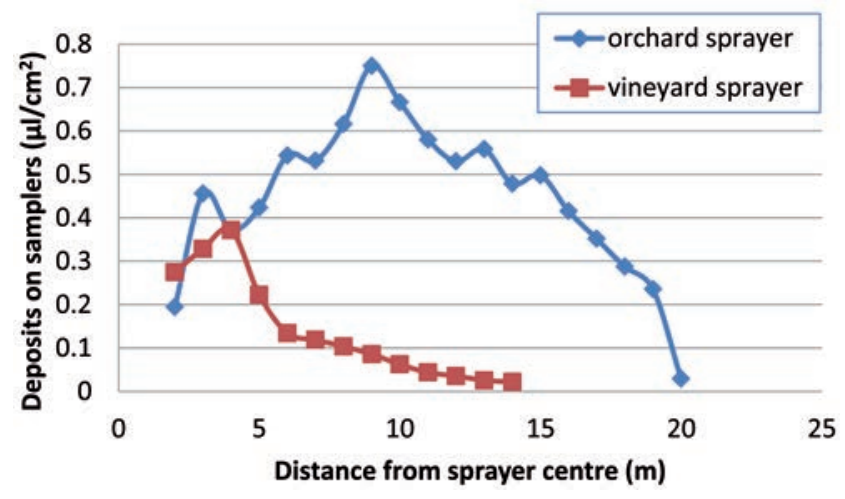

Figure 2. Profiles of spray jet ranges registered for the orchard and for the vineyard sprayers, both equipped with conventional hollow cone nozzles.

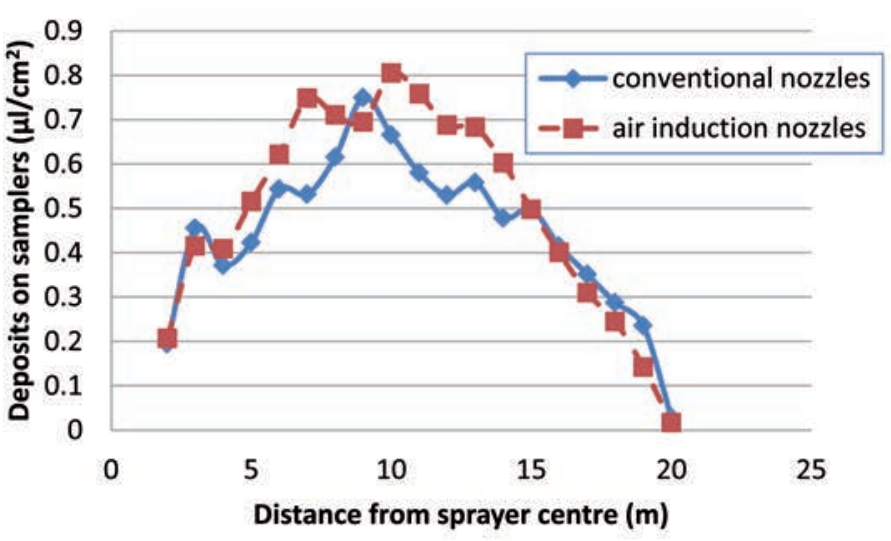

A

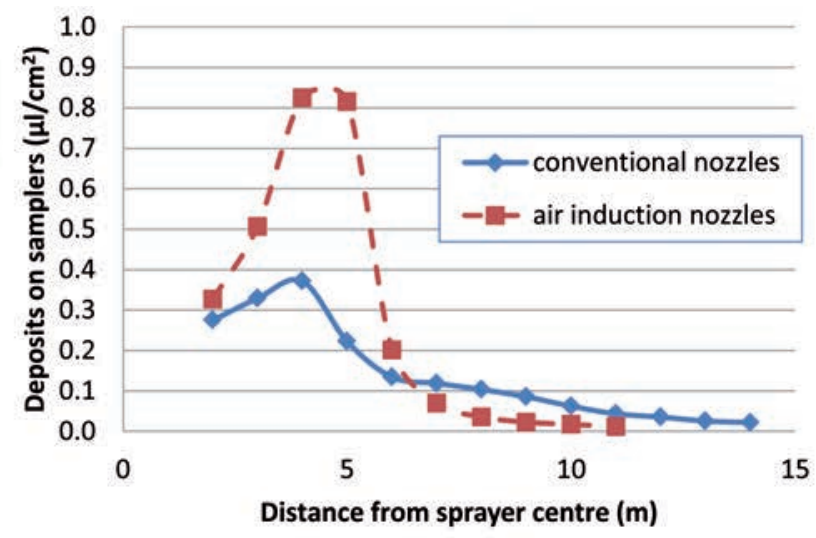

B

Figure 3. Profiles of spray jet range obtained with the orchard sprayer (A) and with the vineyard sprayer (B) in function of the nozzle type employed. 


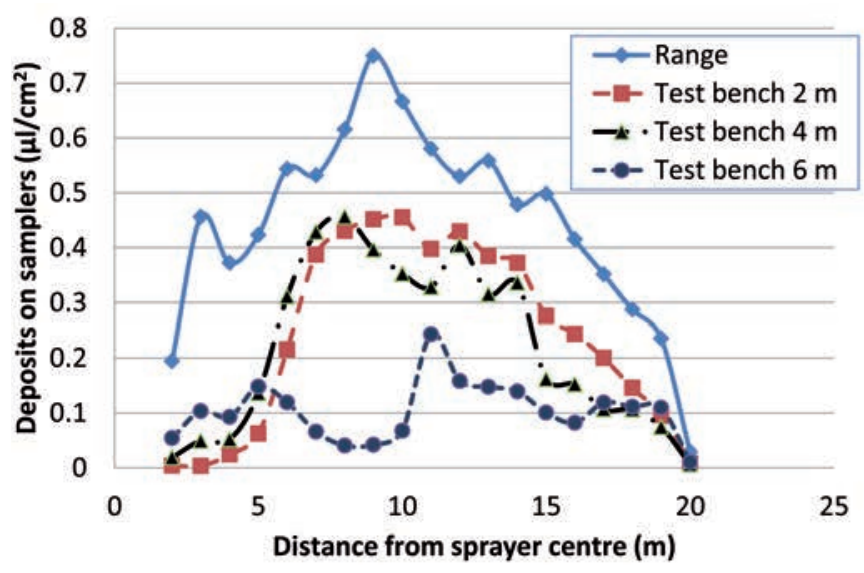

A

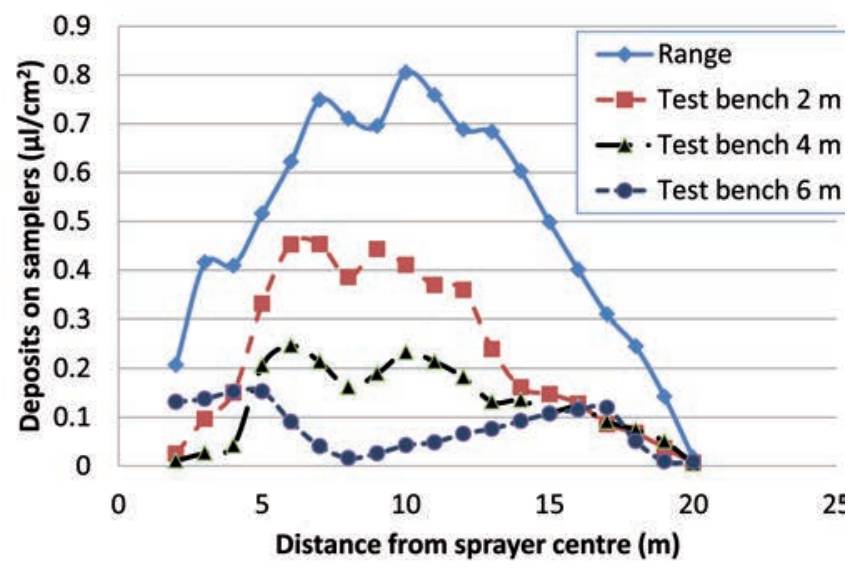

B

Figure 4. Orchard sprayer: profile of the spray jet range and trend of spray deposits detected on samplers positioned along the test benches using conventional hollow cone nozzles $(A)$ and air induction flat fan nozzles (B).

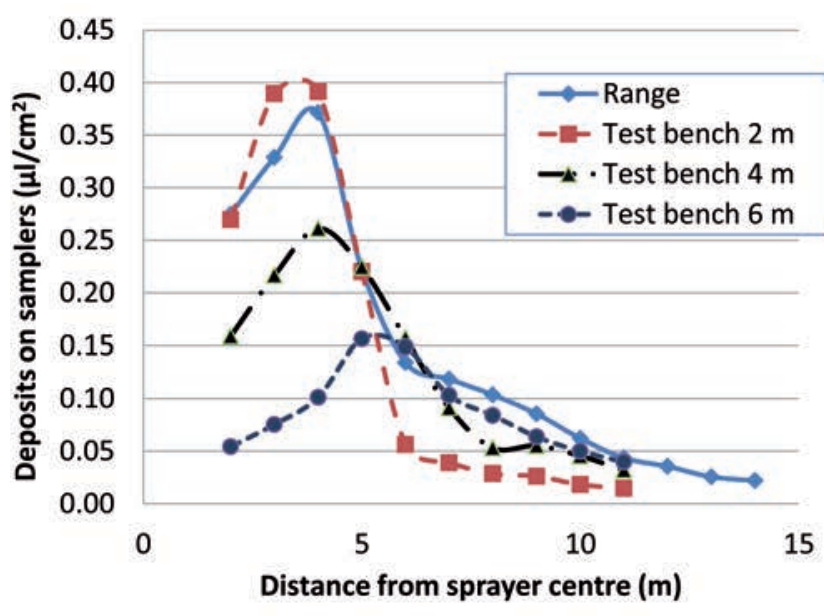

A

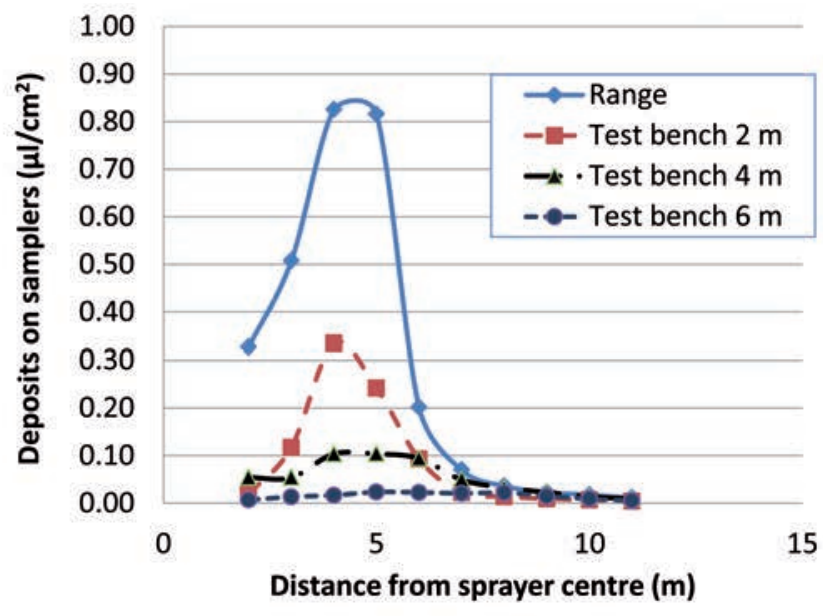

B

Figure 5. Vineyard sprayer: profile of the spray jet range and trend of spray deposits detected on samplers positioned along the test benches using conventional hollow cone nozzles (A) and air induction flat fan nozzles (B).

[3], the index of potential drift DPI was calculated applying the following equation [4]:

$$
\mathrm{DPI}=\mathrm{DP} \times(100-\mathrm{RR}) / 1000
$$

Applying the above mentioned equations to the data collected in the four experimental thesis examined the values of drift potential index (DPI) reported in Table 3 were obtained.

\section{Conclusions}

Comparing the drift potential indexes obtained using the air induction nozzles with respect to those obtained with conventional nozzles it
Table 3. Values of drift potential index calculated for the 4 thesis examined in the experiments.

\begin{tabular}{lcc}
\hline Test & Sprayer and nozmle type & Drift potential index (DPI) \\
1 & Orchard, conventional hollow cone & 254 \\
2 & Orchard, air induction flat fan & 134 \\
\hline 3 & Vineyard, conventional hollow cone & 36 \\
4 & Vineyard, air induction flat fan & 9 \\
\hline
\end{tabular}

was found a reduction of $48 \%$ for the orchard sprayer and a reduction of $75 \%$ for the vineyard one. However, it was noticed that the absolute value of DPI considerably varied in function of the sprayer type (vine- 
yard or orchard) and, therefore, in order to make a consistent classification of sprayers according to drift risk it will be necessary to define a reference sprayer (type and configuration) either for vineyard or for orchard sprayers. Classification in terms of drift reduction (see ISO 22369-1) could be done comparing the DPI of a candidate sprayer with respect to that of the reference sprayer. With the aim to implement the proposed test method in an ISO standard, further tests are on their way aiming at acquiring further data related to different models and configurations of vineyard and orchard air-assisted sprayers.

\section{References}

Balsari, P., Marucco, P., Tamagnone, M., 2007. A test bench for the classification of boom sprayers according to drift risk. Crop protection, $26,1482-1489$.

ISO 22866, 2005. Equipment for crop protection - Methods for field measurement of spray drift.

ISO 22369-1, 2006. Crop protection equipment - Drift classification of spraying equipment - Part 1: Classes. 